\title{
Estrutura social e transmissão intergeracional de status: uma análise hierárquica
}

\author{
Flavia Pereira Xavier* \\ Jorge Alexandre Barbosa Neves**
}

\begin{abstract}
A investigação dos mecanismos de transmissão de status tem correspondido à grande parte dos esforços dos estudos empíricos dos sistemas de estratificação. As diferenças organizacionais encontradas entre setores econômicos e as diferenças regionais inserem-se justamente na dimensão estrutural dos estudos de estratificação. Assim, o principal objetivo deste artigo é investigar em que medida o status ocupacional atual dos indivíduos foi afetado pela sua origem social, considerando-se o momento em que estes indivíduos se inseriram no mercado de trabalho e onde se inseriram. Foram utilizadas as bases da Pesquisa Nacional por Amostra de Domicílios (PNAD) de 1973, 1982, 1988 e 1996 e estimados Modelos Hierárquicos que representam, para os estudos de transmissão intergeracional de status, a possibilidade de um melhor controle de fatores estruturais. Observou-se que a segmentação do mercado de trabalho em diferentes setores econômicos e o nível de crescimento econômico no Brasil em diferentes coortes de entrada dos indivíduos no mercado de trabalho são mecanismos que funcionam como mediadores da transmissão de status, enquanto a segmentação regional não apresentou efeitos significativos.
\end{abstract}

Palavras-chave: Transmissão de status. Regiões do Brasil. Setores econômicos. Crescimento econômico. Coortes de entrada no mercado de trabalho. Modelos hierárquicos lineares.

\section{Introdução}

Investigar os mecanismos de mudança da transmissão de status tem correspondido à grande parte dos esforços dos estudos empíricos dos sistemas de estratificação. A modernização e a urbanização, bem como a expansão do ensino público, incitam os estudiosos a interpretarem a relação entre estes fatores e quais suas consequências na estrutura e no processo de estratificação.
O modelo pelo qual as unidades sociais diferenciam-se e correlacionam-se em termos de poder, riqueza e prestígio, ou seja, a dimensão estrutural da estratificação consiste em grande parte dos esforços da área.

No Brasil, têm sido realizados diversos estudos sobre mobilidade (PASTORE, 1979; PASTORE; SILVA, 2000; RIBEIRO, 2007; SCALON, 1999), bem como sobre realização de status educacional, ocupacional e de rendimentos (FERNANDES, 2005; NEVES

\footnotetext{
* Doutora em Sociologia, professora adjunta do Departamento de Ciências Aplicadas à Educação da Universidade Federal de Minas Gerais - UFMG (flaviapx@ufmg.br).

** Ph.D em Sociologia University of Wisconsin - Madison, professor associado do Departamento de Sociologia e Antropologia da Universidade Federal de Minas Gerais - UFMG, diretor da Faculdade de Filosofia e Ciências Humanas da UFMG (jorgeaneves@gmail.com).
} 
et al., 2007; HELAL, 2008; VILELA, 2008, HALLER; SARAIVA, 1991).

Contudo, ainda poucos se dedicam aos estudos do efeito das estruturas organizacionais no processo de realização de status. As diferenças organizacionais presentes nos setores econômicos, bem como as diferenças de desenvolvimento econômico entre regiões das sociedades modernas, são elementos estruturais que determinam as desigualdades sociais (HELAL, 2008; NEVES et al., 2007).

A ideia central é de que os mercados de trabalho regionais são caracterizados por diferentes processos de recompensas de alocação de status e estes processos estão relacionados ao nível de desenvolvimento econômico de cada região (GRUSKY, 1983). Adicionalmente, as diferenças entre setores econômicos em cada região também interferem no processo de realização de status, bem como o momento da entrada do indivíduo no mercado de trabalho - se em períodos de crescimento, estagnação ou crise econômica.

Foi a partir de 1930 que começou o processo de modernização da sociedade brasileira e a estrutura ocupacional foi transformada pelas mudanças nos planos político e econômico. Mas foi no período pós-Segunda Guerra Mundial até 1960 que o país deu passos mais avançados para a industrialização moderna, na medida em que fortaleceu as políticas protecionistas à indústria doméstica, com apoio à substituição de importações, realizou investimentos estatais na infraestrutura de energia, transportes e produção de insumos básicos, fortaleceu os subsídios e incentivos fiscais, creditícios e cambiais ao investimento privado da indústria e permitiu a entrada massiva de capital estrangeiro na produção de bens manufaturados destinados ao mercado interno, sobretudo a partir de 1950.

Se considerarmos que as mudanças estruturais são importantes para verificar o modo pelo qual a origem social afeta o destino dos indivíduos (seja este educacional, ocupacional ou rendimentos), então estes períodos precisam ser incluídos nas análises das desigualdades. Assim, o principal objetivo deste trabalho é investigar em que medida o status ocupacional atual dos indivíduos é afetado pela sua origem social, levando em conta o momento em que estes indivíduos se inseriram no mercado de trabalho e onde se inseriram (mercados regionais e setoriais). Entendese que a entrada no mercado de trabalho em períodos diferentes em termos de crescimento econômico, bem como as diferenças organizacionais e regionais deste mercado, pode ser compreendida como a estrutura do sistema de status, cujas mudanças afetam o processo de aquisição individual.

Este artigo é composto por nove seções, incluindo esta introdução. As três próximas seções trazem a revisão de um conjunto de teorias que serviram de base para a construção dos objetivos e hipóteses deste trabalho. Posteriormente, são apresentados os dados utilizados, os modelos e as variáveis. Por fim, discutem-se os resultados encontrados e são feitas as considerações finais.

\section{Teoria da industrialização: para uma sociedade meritocrática}

A teoria da industrialização, baseada na tradição funcionalista, estabelece que com o processo de industrialização ocorra uma crescente redução da influência das características atribuídas aos indivíduos (raça, gênero e origem de classe) sobre a realização de status dos indivíduos, ou seja, um crescente predomínio de critérios universalistas no sistema de estratificação social (PARSONS, 1970, 1974; DAVIS; MOORE, 1973; TREIMAN, 1970, 1994; BLAU; DUNCAN, 1967, 1994; DUNCAN, 1966; FEATHERMAN; HAUSER, 1994).

$\mathrm{Na}$ explicação funcionalista, a necessidade universal das desigualdades é aprioristicamente estabelecida, sendo um pré-requisito funcional para o equilíbrio do sistema. O prestígio, o poder e as vantagens são prêmios disputados que só os melhores alcançarão. Então, o funcionamento do sistema depende do reconhecimento legítimo das desigualdades pelos menos privilegiados e recompensados. 
Blau e Duncan (1967, 1994) mudam o foco dos estudos de estratificação, buscando compreender as relações causais da mobilidade. Os autores usam as categorias parsonianas, que caracterizam a sociedade moderna (meritocracia), para desenvolverem a hipótese de que a tendência dessas sociedades é sofrer um aumento da influência do status adquirido sobre o status social do indivíduo e uma queda do status atribuído. ${ }^{1}$ Além disso, o status principal dos indivíduos na sociedade moderna deve-se àquele proveniente de sua posição nas atividades profissionais.

Treiman (1970), em relação à forma pela qual os indivíduos são alocados em diferentes posições, indica que, quanto mais industrializada é uma sociedade, menor a influência do status ocupacional do pai sobre o do filho, menor a influência da educação do pai sobre o alcance educacional do filho, maior o efeito da educação sobre o status ocupacional, mais forte o efeito direto do status ocupacional sobre os rendimentos individuais e, por último, mais fraco o efeito direto da educação sobre os rendimentos. Em suma, menores são as desigualdades de oportunidades.

Complementarmente, os setores econômicos modernos sofrem maior influência de critérios universalistas de seleção, isto é, nesses setores (indústria moderna), as características tais como raça, gênero e origem social possuem menor efeito na realização de status dos indivíduos.

Featherman e Hauser (1994) também consideram que o desenvolvimento econômico, promovido pela industrialização, permite a transição da realização de status baseada na atribuição para aquela baseada em características adquiridas. Em consonância com os outros autores da teoria da industrialização, eles acreditam que as oportunidades para a aprendizagem de habilidades são equalizadas entre as classes sociais à medida que o treinamento ocupacional passa a ser adquirido via escola mais do que via relações de parentesco.
Assim, conforme a teoria da industrialização, foi construída a hipótese 1 , neste estudo, pela qual espera-se que a influência da herança de status, no processo de realização de status ocupacional, diminua com o desenvolvimento econômico e que esta influência seja menor nos setores mais modernos e nas regiões mais desenvolvidas (PARSONS, 1974; TREIMAN, 1970).

\section{Os limites da teoria da industrialização: reprodução e manutenção de status}

Foi Sorokin (2001) o primeiro a analisar a reprodução ou manutenção de status sem a influência da abordagem marxista. Segundo o autor, nas sociedades ocidentais modernas, os grupos ocupacionais estão entrelaçados, fato que contesta a posição dos defensores da teoria da luta de classes, os quais concebem as classes como grupos fechados, esquecendo-se da composição fluida dos atuais grupos ocupacionais. Contudo, apesar da dispersão dos indivíduos em diferentes ocupações, a transmissão "hereditária" persiste, o que significa que, na maioria das vezes, a ocupação do pai continua exercendo forte influência na realização de status do filho. Ainda, Sorokin afirma que a extinção de algumas barreiras à mobilidade vertical, tais como as jurídicas e religiosas, características de um sistema de castas, tende a aumentar esta mobilidade. Entretanto, a remoção dessas barreiras não significa a diminuição absoluta dos obstáculos, mas apenas a substituição de uma série de impedimentos por outros.

Em consonância com Sorokin, a tese da manutenção de status rejeita a visão evolucionária unilinear da teoria da industrialização, uma vez que a expansão escolar, por consequência o aumento do nível educacional, pode substituir o mecanismo de atribuição de status de um caminho indireto mediado pela educação para um caminho mais direto dessa atribuição, ou seja, o efeito direto da herança familiar aumenta, enquanto o efeito mediado pela educação

\footnotetext{
${ }^{1}$ Atribuição (ascription) e aquisição (achievement) são termos introduzidos por Ralph Linton (1959) e referem-se ao termo status, que é um conjunto de direitos e obrigações que especificam as atividades esperadas dos indivíduos incumbidos de uma posição social (HALLER; SARAIVA, 1991).
} 
diminui. Isto se dá porque os recursos, tais como os contatos profissionais dos pais e a riqueza familiar, podem ser usados para que os filhos garantam empregos com maior prestígio, quando a educação não fornece muita informação sobre a produtividade futura (GRUSKY, 1983).

Além do efeito contraditório do declínio da atribuição educacional, a modernização ocupacional é uma variável interveniente adicional que vai mediar o efeito da industrialização nos parâmetros de realização de status. A distribuição ocupacional é determinada por fatores organizacionais e tecnológicos exógenos à oferta educacional (BOUDON, 1981; GRUSKY, 1983).

Ao contrário do que a teoria da industrialização poderia supor, a diminuição da atribuição educacional e a modernização ocupacional podem limitar o desenvolvimento da meritocracia (GRUSKY, 1983).

A formulação de capital cultural e reprodução social de Bourdieu (1998; 2001) é uma ruptura com os pressupostos inerentes à visão de habilidades inatas. A "aptidão" ou "dom", na verdade, são produtos do investimento em capital cultural previamente realizado pela família e reforçado pelo sistema escolar. A apropriação da cultura dominante dá-se pela classe dominante, à medida que os códigos necessários à cultura legitimada são transmitidos pela família. Portanto, o investimento dos pais na carreira dos filhos é um sistema de reprodução. O mercado escolar, por sua vez, sanciona e reproduz a distribuição de capital cultural segundo o legado da família em termos de sucesso escolar. Quando o indivíduo é originário de uma família desprovida de capital econômico e social, mais importante se torna o diploma para ele.

Essa perspectiva corrobora com a argumentação de Collins $(1977 ; 1979),{ }^{2}$ que assinala que a escolaridade não confere ao indivíduo aumento de sua produtividade, mas sim credenciais reconhecidas e legítimas na sociedade. Porém Bourdieu se aprofunda no papel da reprodução social e do capital cultural para as conquistas acadêmicas. Assim, a herança familiar possui grande importância, pois é entendida como uma forma de reprodução de classe.

Conforme a teoria da reprodução social e a tese da manutenção de status, construiuse a hipótese 2 deste artigo, segundo a qual a origem social mantém ou aumenta sua influência no processo de realização de status ocupacional conforme cresce o desenvolvimento econômico (BOURDIEU, 2001; BOWLES; GINTIS, 2000; COLLINS, 1977, 1979; GRUSKY, 1983), bem como seu efeito é igual ou maior nas regiões mais desenvolvidas - regiões urbanizadas com maior expansão do trabalho burocrático (GRUSKY, 1983; GUEST, 2005).

\section{Diferenças entre regiões na transmissão de status: fronteiras de desenvolvimento, industrialização e fluidez social}

A grande hipótese de Turner (1920) é de que as fronteiras norte-americanas foram um local de oportunidades sociais incomuns na maior parte da história do país, ou seja, terras livres significam novas oportunidades. Estas oportunidades que surgem nas fronteiras fortalecem e aumentam a crença na existência de valores democráticos (GUEST, 2005).

Para aqueles que argumentam a favor da tese das fronteiras suscitada por Turner, o desenvolvimento do oeste norte-americano estabeleceu novas oportunidades de mobilidade ascendente sem paralelos em outras nações. Entre os argumentos contrários a esta tese, a principal crítica é de que tal visão romântica do oeste ignora que as grandes oportunidades realmente estão nas cidades, dado que o rápido crescimento da indústria e do comércio nas cidades promove a liberação da população rural excedente não para fixação no oeste. Um terceiro argumento sublinha os erros e acertos da tese das fronteiras e propõe uma hipótese mista. Segundo esta posição

\footnotetext{
2 Destaca-se que Collins desenvolve sua teoria, em oposição ao funcionalismo, baseada nas concepções weberianas de grupos de status, distanciando-se principalmente das visões da reprodução social influenciadas pelo marxismo.
} 
mista, as consequências das oportunidades podem ter sido diferentes nas fronteiras, mas o status final não foi necessariamente diferente. O oeste pode ter selecionado indivíduos bem-sucedidos, tal como os migrantes, mas suas chances podem não ter sido afetadas pela experiência no oeste (GUEST, 2005).

Guest (2005) analisa as diferenças geográficas de nove regiões norte-americanas segundo o padrão de mobilidade em 1800 e $1900 .^{3}$ Conforme os resultados, não há evidências suficientes que confirmem a maioria dos argumentos da tese da fronteira no que diz respeito às oportunidades ocupacionais. A diversidade da estrutura ocupacional na maior parte das áreas urbanas está fortemente relacionada à criação de uma força de trabalho de classe média, que, por seu turno, está relacionada ao maior desenvolvimento e expansão dos trabalhos burocráticos. As taxas de mobilidade ocupacional não variam entre as regiões segundo o sexo ou crescimento populacional. Complementarmente, as maiores taxas de mobilidade foram encontradas nas regiões mais urbanizadas e antigas dos Estados Unidos. A tese de Turner, conforme Guest, pode estar correta não por causa das oportunidades de mobilidade que surgem na fronteira, mas sim pela crença dos novos habitantes de que realmente elas existem.

Outro estudo sobre as diferenças dos efeitos da industrialização entre regiões foi realizado por Grusky (1983). Segundo o autor, o nível de industrialização regional afeta o tamanho dos três parâmetros de realização de status: atribuição educacional (medida pela trajetória entre a origem social e a educação do indivíduo); atribuição de status (medida pela trajetória entre a origem social e o prestígio ocupacional do indivíduo); e aquisição de status (medida pela trajetória entre a educação e o prestígio ocupacional do indivíduo). A ideia central é de que os mercados de trabalho regionais são caracterizados por diferentes processos de recompensas de alocação de status e estes processos estão relacionados ao nível de desenvolvimento econômico de cada região.

Grusky (1983) analisa 12 regiões japonesas com diferentes níveis de industrialização. No primeiro estágio da análise, o autor estima o alcance de status dentro de cada região (trajetória entre origem social e educação e prestígio ocupacional) e, no segundo estágio, correlaciona os parâmetros estimados com variáveis regionais (nível de industrialização e de modernização ocupacional). Os resultados encontrados sugerem que o mercado de trabalho opera no nível local, mesmo em nações altamente industrializadas, como é o caso do Japão. Os retornos ao capital humano estão condicionados ao contexto no qual o indivíduo está localizado. Além disso, segundo o autor, com a industrialização, os efeitos da aquisição de status não aumentam e o processo de atribuição não diminui (este resultado dá suporte parcial à tese da manutenção de status); e a modernização ocupacional também possui impacto moderado nos níveis de atribuição de status. Considerando os achados, o autor conclui que o desenvolvimento da organização burocrática é aparentemente ameaçado pelo fracasso do avanço da industrialização em manter uma contínua expansão de status.

Enquanto Guest (2005) preocupa-se com o nível de industrialização e urbanização de cada região norte-americana no período de expansão da indústria no país, sobretudo, identificando essas regiões como áreas de fronteira de desenvolvimento, Grusky (1983) analisa o Japão da segunda metade do século $X X$, discutindo a fluidez social e o processo de transmissão de status entre as regiões do país, mas sem se dedicar ao teste da hipótese de Turner.

As análises empreendidas pelos autores suscitam a discussão das diferenças regionais ainda em países, tal como o Brasil, que possuem áreas geográficas mais industrializadas e urbanizadas e outras em desenvolvimento.

\footnotetext{
3 As regiões selecionadas no estudo foram aquelas disponíveis no censo norte-americano: New England, Middle Atlantic, South Atlantic, East South Central, West South Central, East North Central, West North Central, Mountain e Pacific.
} 
Neves et al. (2007) analisaram o processo de transmissão intergeracional de status, segundo setores econômicos e áreas geográficas no Brasil. Os resultados apontam que o desenvolvimento econômico afeta igualmente o processo de realização de status, sendo que as regióes e setores econômicos não apresentaram diferenças significativas quanto a este processo. Contudo, há um padrão relativamente estável de reprodução social entre regiões e ao longo do tempo. Especialmente em relação ao teste da teoria das fronteiras em desenvolvimento, os autores ressaltam que não incluíram o setor agrícola na análise, fato que deixa de considerar o peso da estrutura agrária das regiões de fronteira, o que constitui um dos principais motivos das altas taxas de desigualdade e impermeabilidade destas regiões (NEVES et al., 2007).

Conforme a tese das fronteiras em desenvolvimento, hipótese 3 , espera-se que, em regiões em pleno desenvolvimento (fronteiras flexíveis) - Norte e Centro-Oeste -, o efeito da origem social seja menor no processo de realização de status ocupacional do que em regiões já consolidadas - Nordeste, Sul e Sudeste (TURNER, 1920).

\section{Dados}

Os dados a serem utilizados são provenientes da Pesquisa Nacional por Amostra de Domicílios - PNAD de 1973,4 1982, 1988 e 1996. A PNAD é um survey realizado pelo Instituto Brasileiro de Geografia e Estatística - IBGE, formado por um módulo fixo básico e por módulos complementares que variam a cada ano. A pesquisa é realizada em todos os anos não-censitários e constitui-se de amostra estratificada por conglomerados em múltiplos estágios para todo o Brasil, exceto para a região agrária do Norte do país.

Neste trabalho, as PNADs dos referidos anos foram escolhidas por conterem informações sobre mercado de trabalho e escolaridade e, principalmente, pelo módulo de mobilidade social. Este, em especial, fornecerá dados sobre a herança familiar dos indivíduos (escolaridade e ocupação dos pais), cuja importância é imprescindível para os estudos de realização de status.

\section{Modelos e variáveis}

Como estudar o processo de realização de status considerando os mercados regionais e setoriais por coorte uma estrutura e, ao mesmo tempo, levar em conta as características individuais dos sujeitos? Bem, se a pergunta de pesquisa nos parece ter caráter multinível deve ser tratada como tal empiricamente.

Normalmente a solução seria incluir as variáveis de contexto (os mercados regionais e setoriais por coorte) como característica dos indivíduos num único nível para a estimação do resultado do status por MQO. Alternativamente, poderíamos estimar equações por MQO separadas para cada contexto e depois comparar os coeficientes.

Entretanto, quando temos uma pergunta multinível e a tratamos com métodos de nível único (MQO, ANOVA ou equações estruturais), encontramos três dificuldades: viés de agregação; violação da suposição de independência entre variáveis explicativas, ou seja, estimação incorreta dos erros-padrão das estimativas; e heterogeneidade dos coeficientes da regressão (BRYK; RAUDENBUSH, 1992).

Estes problemas podem ser contornados pela aplicação de modelos que não desconsideram a dimensão multinível dos dados. Os modelos hierárquicos ou multiníveis surgem como alternativa aos modelos de MQO, motivados pelo problema enfrentado pelos pesquisadores da área educacional em estimar adequadamente $o$ efeito das escolas sobre o desempenho escolar dos alunos (BRYK; RAUDENBUSH, 1992, p. 5).

Em suma, há três justificativas para o uso de modelos hierárquicos: a empírica; a estatística e da propriedade estrutural dos

\footnotetext{
${ }^{4}$ A PNAD de 1973 não inclui o quesito cor da pele ou raça dos indivíduos, daí a não inclusão desta variável na análise dos efeitos das características adscritivas.
} 
dados; e a teórica. A primeira refere-se à necessidade empírica dessa estrutura ao observarmos a variabilidade da variável resposta por grupos. A maior chave dessa variabilidade é a correlação intraclasse ( $\rho)$. A modelagem hierárquica relaxa o pressuposto da independência dos resíduos e permite estrutura de resíduos correlacionados. Se uma equação por mínimos quadrados ordinários é usada inadequadamente para dados agrupados com erros correlacionados, o resultado dos erros-padrão será menor do que deveria, resultando numa maior chance de cometimento do erro Tipo I. Aqui reside a justificativa estatística para o uso de modelos hierárquicos. Por último, teoricamente levantamos hipóteses compostas por construtos que operam e interagem em múltiplos níveis. Há grande interesse, por exemplo, em saber como características do nível coletivo ou estrutural influenciam o comportamento individual (LUKE, 2004, p.19-23).

\section{Unidade de análise do nível 1}

As unidades de análise do nível 1 (i) são os indivíduos, chefes e cônjuges, entre 15 e 65 anos, ocupados e que não estavam frequentando a escola na semana de referência das PNADs) de 1973, 1982, 1988 e 1996, totalizando cerca de 400 mil unidades.

\section{Construção das unidades de análise do nível 2}

A princípio, considerar os mercados regionais e setoriais por coortes como unidades de análise do nível contextual não é tão óbvio quanto considerar os exemplos clássicos de alunos e escolas, trabalhadores e organizações e, mesmo, escolas e regiões. $\mathrm{Na}$ maioria dos estudos sociológicos, temse buscado tratar a estrutura do mercado de trabalho como um fator importante para alocação ocupacional dos indivíduos e distribuição dos rendimentos do trabalho. Contudo, esta estrutura é inserida no nível individual aplicando os modelos de mínimos quadrados ordinários, sem inquirir se há ou não dependência das observações.

Analisando as diferenças de status ocupacionais pelo modelo hierárquico, re- conhece-se que há variabilidade entre os mercados regionais e setoriais por coortes: pessoas semelhantes quanto às suas características individuais podem possuir status ocupacional distinto por pertencerem a estruturas de mercado diferentes. Observamos, assim, a variabilidade da relação entre status da ocupação atual do indivíduo e status da ocupação do pai por estas estruturas (XAVIER, 2011).

As unidades de nível 2 (j), para representar a estrutura dos mercados regionais e setoriais por coorte, foram criadas pela combinação das regiões do país e setores econômicos por coorte de entrada no mercado de trabalho, totalizando 160 observações neste nível.

Inicialmente, o objetivo era analisar o período entre 1930 (início da modernização do Brasil) e 1989. Entretanto, o número de indivíduos dentro de cada unidade de nível 2 de 1930 a 1949 não era suficiente para o número de parâmetros a serem estimados no modelo. Optou-se, portanto, em analisar o período com tamanho amostral suficiente, mas que ainda se refere ao período da continuação da política de substituição das importações e aceleração do crescimento (1946 a 1964), ampla expansão econômica (1964 a 1982) e recessão econômica até antes do Plano Real (1982 a 1989).

\section{Variáveis}

A variável dependente $\left(Y_{i j}\right)$ é o logaritmo natural do índice de status socioeconômico da ocupação atual do indivíduo (PASTORE, 1979; PASTORE; SILVA, 2000). As variáveis explicativas, separadas por nível, são apresentadas nos Quadros 1 e 2.

A média da variação do PIB per capita por coorte de entrada no mercado de trabaIho mede a taxa de crescimento econômico, enquanto a média do PIB per capita mede o tamanho ou nível desse crescimento. Em termos restritivos, a medida mais geral de desenvolvimento econômico é o aumento do PIB, porque ele reflete a expansão geral da produtividade do país. Alternativamente, há o Índice de Desenvolvimento Humano, que é muito mais que um índice de desenvolvimento econômico como permitem 
QUADRO 1

Variáveis de nível 1

\begin{tabular}{|llll|}
\hline Variáveis de nível 1 & Tipo de variável & Descrição & Tipo de centralização (1) \\
\hline Sexo & Binária & Homens =1 e Mulheres =o & Não centralizada \\
\hline $1982(2)$ & Binária & Entrevistados em 1982 & Não centralizada \\
\hline $1988(2)$ & Binária & Entrevistados em 1988 & Não centralizada \\
\hline $1996(2)$ & Binária & Entrevistados em 1996 & Não centralizada \\
\hline Experiência & Discreta & $\begin{array}{l}\text { Idade em que o indivíduo começou a } \\
\text { trabalhar subtraída da idade (em anos) }\end{array}$ & $\begin{array}{l}\text { Centralizada pela média do } \\
\text { grupo }\end{array}$ \\
\hline $\begin{array}{l}\text { Experiência ao } \\
\text { quadrado }\end{array}$ & Discreta & $\begin{array}{l}\text { Experiência elevada ao quadrado } \\
\text { (anos ao quadrado) }\end{array}$ & $\begin{array}{l}\text { Centralizada pela média do } \\
\text { grupo }\end{array}$ \\
\hline Educação & Discreta & $\begin{array}{l}\text { Medida em número de séries } \\
\text { concluídas }\end{array}$ & $\begin{array}{l}\text { Centralizada pela média do } \\
\text { grupo }\end{array}$ \\
\hline $\begin{array}{l}\text { Status da ocupação } \\
\text { do pai }\end{array}$ & Contínua & $\begin{array}{l}\text { Logaritmo natural do índice de status } \\
\text { socioeconômico da ocupação do pai } \\
\text { do indivíduo }\end{array}$ & $\begin{array}{l}\text { Centralizada pela média do } \\
\text { grupo }\end{array}$ \\
\hline $\begin{array}{l}\text { Status da primeira } \\
\text { ocupação }\end{array}$ & Contínua & $\begin{array}{l}\text { Logaritmo natural do índice de status } \\
\text { socioeconômico da primeira ocupação } \\
\text { do indivíduo }\end{array}$ & $\begin{array}{l}\text { Centralizada pela média do } \\
\text { grupo }\end{array}$ \\
\hline
\end{tabular}

(1) Sobre centralização das variáveis nos dois níveis ver Bryk e Raudenbush (1992).

(2) Categoria de referência: entrevistados em 1973.

QUADRO 2

Variáveis de nível 2

\begin{tabular}{|c|c|c|c|}
\hline Variáveis de nível 2 & Tipo de variável & Descrição & Tipo de centralização \\
\hline $\begin{array}{l}\text { Média da variação anual do } \\
\text { PIB por coorte }\end{array}$ & Contínua & $\begin{array}{l}\text { Média da variação anual do Produto } \\
\text { Interno Bruto deflacionado referente } \\
\text { a cada coorte de entrada no mercado } \\
\text { de trabalho }\end{array}$ & $\begin{array}{l}\text { Centralizada pela grande } \\
\text { média }\end{array}$ \\
\hline $\begin{array}{l}\text { Média do PIB per capita por } \\
\text { coorte }\end{array}$ & Contínua & $\begin{array}{l}\text { Média do Produto Interno Bruto per } \\
\text { capita a preços correntes referente a } \\
\text { cada coorte de entrada no mercado } \\
\text { de trabalho }\end{array}$ & $\begin{array}{l}\text { Centralizada pela grande } \\
\text { média }\end{array}$ \\
\hline $\begin{array}{l}\text { Média da taxa de } \\
\text { urbanização por coorte }\end{array}$ & Contínua & $\begin{array}{l}\text { Média do total da população urbana } \\
\text { dividida pelo total da população } \\
\text { residente no país multiplicado por } \\
\text { cem em cada coorte de entrada no } \\
\text { mercado de trabalho }\end{array}$ & $\begin{array}{l}\text { Centralizada pela grande } \\
\text { média }\end{array}$ \\
\hline Região Sul (1) & Binária & $\begin{array}{l}\text { Paraná, Santa Catarina e Rio Grande } \\
\text { do Sul }\end{array}$ & Não centralizada \\
\hline Região Nordeste (1) & Binária & $\begin{array}{l}\text { Bahia, Pernambuco, Rio Grande do } \\
\text { Norte, Paraíba, Alagoas, Maranhão, } \\
\text { Ceará, Piauí e Sergipe }\end{array}$ & Não centralizada \\
\hline Região Centro-Oeste (1) & Binária & $\begin{array}{l}\text { Mato Grosso (inclui Mato Grosso e } \\
\text { Mato Grosso do Sul), Goiás (inclui } \\
\text { Goiás e Tocantins) e Distrito Federal }\end{array}$ & Não centralizada \\
\hline Região Norte (1) & Binária & $\begin{array}{l}\text { Pará, Acre, Amazonas, Rondônia, } \\
\text { Roraima e Amapá }\end{array}$ & Não centralizada \\
\hline Setor tradicional (2) & Binária & $\begin{array}{l}\text { Inclui todas as atividades agrícolas, } \\
\text { pecuárias e extrativismo }\end{array}$ & Não centralizada \\
\hline Setor flexível (2) & Binária & $\begin{array}{l}\text { Inclui todas as atividades de prestação } \\
\text { de serviços, comércio, transporte e } \\
\text { social }\end{array}$ & Não centralizada \\
\hline Setor público (2) & Binária & $\begin{array}{l}\text { Atividades da administração pública } \\
\text { e defesa }\end{array}$ & Não centralizada \\
\hline
\end{tabular}

(1) Categoria de referência: Região Sudeste (região mais industrializada): Rio de Janeiro (inclui o Estado da Guanabara de 1973), São Paulo, Minas Gerais e Espírito Santo.

(2) Categoria de referência: setor rígido ou industrial (inclui todas as atividades industriais). 
os dados de renda per capita ou PIB, pois inclui, além da renda per capita, a longevidade e a alfabetização, buscando refletir o bem-estar social.

Para Bresser-Pereira (2007), o IDH é um índice muito mais de desenvolvimento em geral do que de crescimento econômico. Para além da polêmica sobre a equivalência ou não de desenvolvimento econômico e crescimento econômico, a melhor maneira de medi-lo é pelo aumento da renda per capita. O IDH não pode ser usado para medir a taxa de desenvolvimento econômico.

Não incluímos o IDH porque os dados do índice estão disponíveis apenas decenalmente a partir de 1970. Dessa forma, não seria possível analisar as coortes de entrada no mercado de trabalho aqui propostas (1950 a 1989).

Duas variáveis importantes não foram incluídas na análise de transmissão de status: cor ou raça e migração. Mesmo que as variáveis adscritivas não sejam o teste central deste estudo, elas deveriam estar incluídas porque explicam parte importante da variância no status ocupacional e possibilitam uma medida líquida das variáveis de teste mais confiáveis. Entretanto, antes da década de 1980, os questionários do IBGE não incluíam os quesitos cor ou raça do indivíduo.

Migração, juntamente com educação e experiência, é considerada recurso fundamental de capital humano (SCHULTZ, 1973). A migração significa possibilidades de ascensão social e, dessa maneira, à medida que os indivíduos percebem esse fato, a movimentação geográfica de mão de obra é substancialmente ampliada (PASTORE, 1979).

Na base de 1973, a informação disponível sobre migração é a região do país de nascimento do indivíduo, o que não fornece uma medida adequada de recursos e motivação dos indivíduos quanto às possibilidades de mobilidade social ascendente. Já a base de 1983 não contém esta variável.

\section{Especificação dos modelos}

Dois modelos foram estimados: o modelo de análise de variância (ANOVA) ou modelo nulo, que permite verificar se há variação entre os mercados regionais e setoriais segundo coortes de entrada no mercado de trabalho e, portanto, se é possível estimar o modelo hierárquico; e o modelo do intercepto e da inclinação como resposta (intercept and slopes-as-outcomes model), com variáveis de níveis 1 e 2 , cujo interesse está na interação entre mercados e status da ocupação do pai.

Modelo I - ANOVA (modelo nulo)

$\operatorname{Ln} Y_{i j}=\beta_{0 j}+r_{i j}$

$B_{0 j}=\mathrm{Y}_{00}+\mathrm{u}_{0 j}$

Modelo II - modelo do intercepto e da inclinação como resposta

$\operatorname{Ln} Y_{i j}=\beta_{\text {oj }}+\beta_{1}$ (sexo) $+\beta_{2}(1982)+$ $\beta_{3}(1988)+\beta_{4}(1996)+\beta_{5}$ (experiência) $+\beta_{6}$ (experiência ao quadrado) + $\beta_{7}$ (educação) $+\beta_{8}$ (In do status da ocupação do pai $)_{j}+\beta_{9}$ (In do status da primeira ocupaçao) $+\mathrm{r}_{\mathrm{ij}}$

$B_{0 j}=\gamma_{00}+\gamma_{01}$ (média da variação do PIB anual) $+Y_{02}$ (média do PIB per capita) + $Y_{03}$ (média da taxa de urbanização) $+Y_{04}$ (sul) $+Y_{05}$ (nordeste) $+Y_{06}$ (centro-oeste) $+Y_{07}$ (norte) $+Y_{08}$ (tradicional) $+Y_{09}$ (flexível) $+\mathrm{Y}_{010}$ (público) $+\mathrm{u}_{0 \mathrm{j}}$

$B_{8 j}=\gamma_{80}+\gamma_{81}$ (média da variação do PIB anual) $+Y_{82}$ (média do PIB per capita) + Y83 (média da taxa de urbanização) $+Y_{84}$ (sul) $+Y_{85}$ (nordeste) $+Y_{86}$ (centro-oeste) $+\gamma_{87}$ (norte) $+\gamma_{88}$ (tradicional) $+Y_{89}$ (flexível) $+Y_{810}$ (público) $+\mathrm{u}_{8 j}$ onde:

$\operatorname{Ln} Y_{i j}=$ logaritmo do status da ocupação atual do i-ésimo indivíduo do j-ésimo mercado regional e setorial por coorte;

$i=1,2, \ldots, \mathrm{n}_{\mathrm{j}}$ é o número de indivíduos do j-ésimo mercado regional e setorial por coorte;

$j=1,2, \ldots, \mathrm{l}$, onde lé o número de mercados regionais e setoriais por coorte;

$r_{i j}=$ efeito aleatório do nível 1 (resíduos relativos aos indivíduos);

$u_{0 j}$ e $u_{8 j}=$ efeito aleatório do nível 2 (resíduos relativos aos mercados); 


\section{Resultados}

Pelo modelo nulo, observa-se que a média do status da ocupação atual dos indivíduos é igual a 13,48 pontos ${ }^{5}$ (Tabela 1). Os efeitos aleatórios (Tabela 2) fornecem as estimativas dos componentes de variância. No nível 1 , a Vârrij $=\sigma 2=0,424$ e, no nível 2, a variância do status ocupacional médio $\left(\beta_{0 j}\right)$ é $\tau_{00}=0,473$. A proporção da variância em $Y$ entre os mercados é representada pela correlação intraclasse $(\rho)$ e pode ser calculada da seguinte forma:

$\rho=\tau 00 \tau 00+\sigma 2$

Pelo cálculo de $\rho$, verifica-se que $52,73 \%$ do total da variação do status da ocupação atual dos indivíduos ocorre entre as unidades de nível 2 (mercados regionais e setoriais por coorte).

Em outras palavras, a variação total observada entre o status do indivíduo foi igual a $0,897\left(\tau_{00}+\sigma 2\right)$, sendo que $57,73 \%$ do total da variação deve-se à diferença das médias entre os mercados e $47,27 \%$ são explicados pela variação do status dentro dos mercados. Este resultado confirma que há variabilidade entre grupos e que podemos prosseguir com o modelo hierárquico, isto é, há justificativa empírica para a aplicação do modelo hierárquico.

Pela significância de $\tau_{00}$, concluímos que todos os mercados regionais e setoriais por coorte possuem médias diferentes (rejeitamos a hipótese nula de que todas as unidades de nível 2 assumem a mesma média, $\mathrm{H}_{0}: \tau_{00}=0$ ).

As Tabelas 3 e 4 apresentam os resultados do modelo IV. Verifica-se que nem a taxa de urbanização nem as regiões do país possuem efeito significativo sobre a média do status da ocupação atual do indivíduo $\left(Y_{03}, Y_{04}, Y_{05}, Y_{06}\right.$ e $\left.Y_{07}\right)$. Já a média da variação do PIB possui efeito negativo, enquanto a média do PIB per capita tem efeito positivo $\left(Y_{01}\right.$ e $\left.Y_{02}\right)$ : a variação de $1 \%$ no PIB diminui em $4,5 \%$ a média do status atual e o aumento de $\mathrm{R} \$ 1,00$ no PIB per capita eleva em $0,00004 \%$ o status. Os setores econômicos são todos significantes $\left(Y_{08}, Y_{09}\right.$ e $\left.Y_{010}\right)$ : os indivíduos do setor tradicional possuem a média do status $45,49 \%$ menor do que a média dos indivíduos do setor industrial; os indivíduos do setor flexível possuem a média $22,85 \%$ maior do que a dos indivíduos do setor industrial; e os da administração pública

TABELA 1

Efeito fixo do Modelo I (ANOVA)

\begin{tabular}{lccccc}
\hline Efeito fixo & Coeficiente & Erro-padrão & Razão T & gl & Valor $\mathbf{p}$ \\
\hline Intercepto $Y_{00}$ & 2,601 & 0,054 & 47,93 & 159 & 0,000 \\
\hline
\end{tabular}

Fonte: Elaboração própria a partir dos dados das PNADs de 1973, 1982, 1988 e 1996.

TABELA 2

Efeitos aleatórios do Modelo I (ANOVA)

\begin{tabular}{lccccc}
\hline Efeitos aleatórios & Desvio-padrão & Componentes da variância & gl & Qui-quadrado & Valor $\mathbf{p}$ \\
\hline Intercepto, $\mathrm{u}_{0 \mathrm{j}}$ & 0,688 & 0,473 & 159 & 413020,65 & 0,000 \\
Nível 1, $\mathrm{r}_{\mathrm{ij}}$ & 0,651 & 0,424 & & & \\
\hline
\end{tabular}

Fonte: Elaboração própria a partir dos dados das PNADs de 1973, 1982, 1988 e 1996.

\footnotetext{
5 Para determinar a média do status, dado que a variável dependente é o logarítmo do status, basta realizar o seguinte cálculo: $\exp \left(\mathrm{Y}_{00}\right)$ (GUJARATI, 2000, p.172-173).
} 
TABELA 3

Efeitos fixos do Modelo II

\begin{tabular}{|c|c|c|c|c|c|}
\hline Efeitos fixos & Coeficiente & Erro-padrão & Razão T & gl & Valor $p$ \\
\hline \multicolumn{6}{|l|}{ Intercepto $\beta_{0}$} \\
\hline Intercepto $Y_{00}$ & 1,580 & 0,046 & 34,292 & 149 & 0,000 \\
\hline Média da variação anual do PIB $Y_{01}$ & $-0,046$ & 0,004 & $-12,513$ & 149 & 0,000 \\
\hline Média do PIB per capita $Y_{02}$ & 0,00004 & 0,000 & 1,941 & 149 & 0,052 \\
\hline Média da taxa de urbanização $Y_{03}$ & $-0,005$ & 0,006 & $-0,829$ & 149 & 0,407 \\
\hline Sul $Y_{04}$ & 0,014 & 0,040 & $-1,573$ & 149 & 0,730 \\
\hline Nordeste $Y_{05}$ & $-0,063$ & 0,040 & $-1,573$ & 149 & 0,116 \\
\hline Centro-Oeste $Y_{06}$ & $-0,009$ & 0,042 & $-0,221$ & 149 & 0,825 \\
\hline Norte $Y_{07}$ & $-0,019$ & 0,039 & $-0,500$ & 149 & 0,616 \\
\hline Setor tradicional $Y_{08}$ & $-0,607$ & 0,463 & $-13,115$ & 149 & 0,000 \\
\hline Setor flexível Yo9 & 0,206 & 0,030 & 6,879 & 149 & 0,000 \\
\hline Setor público Yo10 & 0,524 & 16,231 & 16,231 & 149 & 0,000 \\
\hline \multicolumn{6}{|l|}{ Sexo $\beta_{1}$} \\
\hline Intercepto $Y_{10}$ & 0,214 & 0,014 & 15,60 & 400067 & 0,000 \\
\hline \multicolumn{6}{|l|}{$1982 \beta_{2}$} \\
\hline Intercepto $Y_{20}$ & 0,015 & 0,016 & 0,96 & 400067 & 0,337 \\
\hline \multicolumn{6}{|l|}{$1988 \beta_{3}$} \\
\hline Intercepto $Y_{30}$ & 0,061 & 0,015 & 4,17 & 400067 & 0,000 \\
\hline \multicolumn{6}{|l|}{$1996 \beta_{4}$} \\
\hline Intercepto $Y_{40}$ & 0,865 & 0,028 & 30,789 & 400067 & 0,000 \\
\hline \multicolumn{6}{|l|}{ Experiência $\beta_{5}$} \\
\hline Intercepto $Y_{50}$ & 0,007 & 0,0004 & 17,00 & 400067 & 0,000 \\
\hline \multicolumn{6}{|l|}{ Experiência ao quadrado $\beta_{6}$} \\
\hline Intercepto $Y_{60}$ & $-0,0002$ & 0,00002 & $-12,61$ & 400067 & 0,000 \\
\hline \multicolumn{6}{|l|}{ Educação $\beta_{7}$} \\
\hline Intercepto $Y_{70}$ & 0,0592 & 0,00214 & 27,64 & 400067 & 0,000 \\
\hline \multicolumn{6}{|l|}{ Status do pai $\beta_{8}$} \\
\hline Intercepto $Y_{80}$ & 0,114 & 0,013 & 8,711 & 149 & 0,000 \\
\hline Média da variação anual do PIB $Y_{81}$ & $-0,00182$ & 0,002 & $-1,083$ & 149 & 0,279 \\
\hline Média do PIB per capita $\bigvee_{82}$ & $-0,00014$ & 0,00001 & $-2,794$ & 149 & 0,006 \\
\hline Média da taxa de urbanização $\bigvee_{83}$ & 0,001 & 0,002 & 0,476 & 149 & 0,634 \\
\hline Sul $Y_{84}$ & $-0,017$ & 0,013 & $-1,300$ & 149 & 0,194 \\
\hline Nordeste $Y_{85}$ & $-0,010$ & 0,012 & $-0,807$ & 149 & 0,420 \\
\hline Centro-Oeste $Y_{86}$ & $-0,011$ & 0,012 & $-0,934$ & 149 & 0,351 \\
\hline Norte $Y_{87}$ & $-0,002$ & 0,013 & $-0,171$ & 149 & 0,865 \\
\hline Setor tradicional $Y_{88}$ & 0,039 & 0,013 & 2,950 & 149 & 0,004 \\
\hline Setor flexível Y89 & $-0,033$ & 0,010 & $-3,426$ & 149 & 0,001 \\
\hline Setor público $Y_{810}$ & $-0,055$ & 0,011 & $-5,130$ & 149 & 0,001 \\
\hline \multicolumn{6}{|l|}{ Status da primeira ocupação $\beta_{9}$} \\
\hline Intercepto $Y_{90}$ & 0,32 & 0,012 & 26,75 & 400067 & 0,000 \\
\hline
\end{tabular}

Fonte: Elaboração própria a partir dos dados das PNADs de 1973, 1982, 1988 e 1996. 
TABELA 4

Efeitos aleatórios do Modelo II

\begin{tabular}{lccccc}
\hline Efeitos aleatórios & Desvio-padrão & Componentes da variância & gl & Qui-quadrado & Valor $\mathbf{p}$ \\
\hline Intercepto, $\mathrm{u}_{0 \mathrm{j}}$ & 0,169 & 0,02867 & 149 & 58198,67 & 0,000 \\
Status do pai, $\mathrm{u}_{8 \mathrm{j}}$ & 0,0458 & 0,0021 & 149 & 1380,14 & 0,000 \\
Nível 1, $\mathrm{r}_{\mathrm{ij}}$ & 0,40291 & 0,16234 & & & \\
\hline
\end{tabular}

Fonte: Elaboração própria a partir dos dados das PNADs de 1973, 1982, 1988 e 1996.

possuem a média $68,87 \%$ maior do que a daqueles do setor industrial. ${ }^{6}$

Nota-se também que não há interação entre o status do pai e a média da variação do PIB, a taxa de urbanização e as regiões do país sobre o status da ocupação atual do indivíduo $\left(Y_{81}, Y_{83}, Y_{84}, Y_{85}, Y_{86}\right.$ e $\left.Y_{87}\right)$. Já a média do PIB per capita $\left(\gamma_{82}\right)$ diminui o efeito do status do pai sobre o status da ocupação atual do indivíduo em $0,18 \%$. O setor tradicional aumenta o efeito do status do pai sobre o status da ocupação atual do indivíduo em $3,95 \%\left(\gamma_{88}\right)$, enquanto os setores flexível e público $\left(\mathrm{Y}_{88}\right.$ e $\left.\mathrm{Y}_{810}\right)$ diminuem o efeito do status do pai sobre o status da ocupação atual do indivíduo em $3,25 \%$ e $5,36 \%$, respectivamente, comparados ao setor industrial.

A tese da industrialização prevê um processo de "desestratificação" com o avanço do desenvolvimento econômico: diminuição da influência da herança de status e aumento do efeito da educação sobre o status ocupacional corrente do indivíduo. Pelos resultados ora apresentados, temse a evidência de que a média do PIB per capita (nível do crescimento econômico) diminui o efeito da origem social (status do pai) sobre o destino ocupacional (status da ocupação atual do indivíduo), influência esta que ocorre independentemente das regiões do país, dos setores econômicos, da taxa de urbanização e da variação do PIB. Nesse sentido, não foi confirmada completamente a hipótese 1: por um lado, como previsto pela teoria, a herança de status diminui seu efeito sobre o status corrente com o processo de industrialização e os setores mais modernos da economia contribuem para a diminuição do processo de alocação de status, mas, por outro lado, as regiões mais industrializadas (Sul e Sudeste) não diminuíram o efeito de transmissão de status.

O nosso achado vai de encontro aos de outras pesquisas. Bills e Haller (1984) não encontraram nenhum padrão significativo do PIB no efeito do status do pai. Neves et al. (2007) também não observaram nenhum padrão sistemático do nível do crescimento econômico (PIB per capita), o que levou os autores a concluírem que não há decréscimo, ao longo do tempo, dos padrões de transmissão de status. Um motivo para que nossos resultados em relação ao impacto do desenvolvimento econômico sobre a transmissão de status tenham se dado em outro sentido pode estar na inclusão do nível do crescimento econômico e da sua taxa de variação por coortes. Assim, percebemos o impacto deste fator segundo as gerações, traduzindo melhor as oportunidades da estrutura ocupacional.

Já a hipótese 2 afirma que não há perda da influência da origem social no processo de realização de status ocupacional com o avanço do desenvolvimento econômico e que o efeito da origem social não difere nas regiões mais urbanizadas e com maior expansão do trabalho burocrático. Esta hipótese também foi parcialmente confirmada. Por um lado, ao contrário do esperado, não se observou nenhuma

\footnotetext{
6 O efeito para toda variável de nível 2 do modelo, W, é dado por $\operatorname{Exp}\left(\mathrm{Y}_{\mathrm{gs}} \mathrm{s}^{-1}\right)^{*} 100$. O efeito de todo $\mathrm{X}$, exceto para o $\mathrm{Ln}$ do status do pai e Ln do status da primeira ocupação, é dado por $\operatorname{Exp}\left(\beta_{\mathrm{qj}}{ }^{-1}\right)^{*} 100$. O efeito dos interceptos é dado por $\operatorname{Exp}\left(\mathrm{Y}_{00}\right)$. O efeito do Ln do status do pai e do Ln do status da primeira ocupação é interpretado como: quando $\mathrm{X}$ aumenta em $1 \%, Y_{i j}$ aumenta em $\beta \%$ (GUJARATI, 2000, p. 172-174).
} 
constância ou aumento no peso da origem social, pelo contrário, foi verificada uma queda, indicando uma estrutura ocupacional menos rígida com o aumento do nível de desenvolvimento. Por outro lado, nem a taxa de urbanização nem as regiões do país apresentaram efeito significativo sobre o impacto da origem no status corrente do indivíduo, confirmando o previsto pelas teorias da manutenção de status e da reprodução social.

A tese das fronteiras flexíveis, defendida por Turner (1920) para os Estados Unidos (hipótese 3), não foi confirmada para o caso brasileiro, uma vez que não foi encontrado efeito significativo das regiões em pleno desenvolvimento (Centro-Oeste e Norte) sobre o nível de transmissão de status.

Neves et al. (2007) também não encontram fortes evidências que dessem suporte à tese das fronteiras em desenvolvimento, entretanto, como os próprios autores ressaltam, o seu estudo engloba o Norte, mas não incluiu o meio rural do Brasil. No nosso trabalho, torna-se mais claro que a transmissão intergeracional de status não sofre impacto das regiões em pleno desenvolvimento porque incluímos as cinco regiões do país.

\section{Considerações finais}

A rápida transição de uma sociedade agrícola para uma industrial modificou profundamente a estrutura ocupacional brasileira, aumentando o tamanho das classes manuais urbanas e, consequentemente, favorecendo as altas taxas de mobilidade total.

Pastore (1979) afirma que os altos níveis de desigualdade na década de 1970 não eram incompatíveis com a taxa de mobilidade brasileira, uma vez que esta era causada pelas rápidas mudanças estruturais do país: transformações econômicas, tecnológicas e demográficas. Os efeitos dessas mudanças verificadas entre 1973 e 1988 consistem na ampliação da ascensão social, especialmente, na base da pirâmide social e na predominância da mobilidade estrutural e numa influência relativamente pequena da herança social paterna.

Contudo, se a transição para uma economia industrial e de serviços possibi- litou aumento da mobilidade estrutural e ascendente entre 1973 e 1988, relativamente o mesmo não ocorreu entre 1988 e 1996 (PASTORE; SILVA, 2000).

Os padrões encontrados por Ribeiro (2007) entre 1973 e 1996 indicam menos mobilidade ascendente, menos mobilidade estrutural (efeito estrutural gerado pela rápida industrialização no país) e mais fluidez (menores desigualdades de oportunidades). A interpretação deste padrão foi feita pelo autor como tendência de uma menor transmissão intergeracional de vantagens e desvantagens e, consequentemente, de chances de ascensão mais condicionadas pelas habilidades individuais do que pela origem social, isto é, as desigualdades de oportunidades têm diminuído no Brasil, uma vez que um percentual maior da população passou a ter mais acesso à educação média e superior e as classes de origem possuem menos importância na determinação das chances de mobilidade.

Se, por um lado, tem-se aumento das desigualdades de renda, por outro, não houve crescimento das desigualdades ocupacionais em geral, medidas pela fluidez social. Mas vale ressaltar que as desigualdades de oportunidades ocupacionais aplicam-se à população ocupada e diferem segundo os grupos por sexo e cor.

Todos estes estudos indicam que o efeito da herança de status depende de fatores estruturais importantes que variam ao longo do processo de industrialização no Brasil. Assim, partindo do pressuposto de que a entrada no mercado de trabalho em períodos diferentes em termos de crescimento econômico, bem como as diferenças organizacionais e regionais deste mercado, pode ser compreendida como a estrutura do sistema de status, cujas mudanças afetam o processo de aquisição individual, o presente estudo buscou investigar como se dá a transmissão intergeracional de status dentro dessa estrutura de posições.

Foram propostas três hipóteses de trabalho, baseadas na diversa literatura sobre o tema. A primeira hipótese, baseada na teoria da industrialização, previa uma diminuição da transmissão de status com o crescimento econômico e uma menor transmissão nas 
regiões mais industrializadas e nos setores econômicos mais modernos. Em oposição, baseada na teoria da reprodução social e na tese da manutenção de status, a segunda hipótese previa que a transmissão intergeracional de status não seria menor com o crescimento econômico e nem nas regiões mais industrializadas e urbanizadas, respectivamente. A terceira hipótese, baseada na tese das fronteiras flexíveis, previa menor transmissão de status nas regiões Centro-Oeste e Norte.

Foram encontradas evidências que confirmam parcialmente a primeira hipótese, já que o nível de crescimento econômico e os setores mais modernos diminuem o processo de transmissão intergeracional de status ocupacional.

A segunda hipótese também foi parcialmente confirmada, uma vez que nem a taxa de urbanização nem as regiões do país apresentaram impacto significativo sobre o efeito da origem no status corrente do indivíduo.

A terceira hipótese, por sua vez, não foi confirmada, pois não foi observado efeito significativo das regiões em pleno desenvolvimento (Centro-Oeste e Norte).

Em suma, o crescimento econômico reduz a transmissão de status: não há diferença entre as regiões do país em termos de transmissão de status, mas há entre os diferentes setores. Os setores flexível e público apresentam menor reprodução social quando comparados ao setor industrial. Por sua vez, o nível do crescimento econômico, calculado pela média do PIB per capita nas coortes de entrada no mercado de trabalho, está negativamente correlacionado à transmissão de status, mas esta não se correlaciona ao desempenho do crescimento, dado pela média da variação anual do PIB nas coortes.

Este fato pode estar relacionado ao aumento das oportunidades ocupacionais pela transformação da estrutura do mercado de trabalho e pela importância das habilidades adquiridas pelo indivíduo, tal como a educação, na determinação do alcance ocupacional.

Ressalta-se que esses padrões de transmissão intergeracional de status demonstram efeitos setoriais, mas não regionais no período de 1950 a 1989. Não é possível inferir uma tendência para as décadas de 1990 e 2000, quando assistimos a um período de hiperinflação e estagnação (de 1986 a 1994), de estabilidade monetária e estagnação (de 1995 a 2003) e depois de recuperação do crescimento econômico (após 2003).

Grande debate tem sido feito sobre as desigualdades regionais no Brasil, no que se refere seja às diferenças inter-regionais de renda per capita seja às diferenças da mobilidade ocupacional. Pastore (1979) entende que o Brasil possui disparidades regionais, porque as regiões possuem diferentes estruturas de oportunidades e, portanto, níveis distintos de mobilidade inter e intrageracional. $\mathrm{O}$ autor conclui que as taxas de mobilidade social no Brasil, em 1973, estavam bastante associadas ao nível de urbanização e industrialização das regiões de destino dos indivíduos.

As diferenças regionais no processo de realização de status ocupacional dos indivíduos não são captadas pelo presente estudo, porque são obtidas pelo PIB per capita e pelos setores da economia. Então, as diferenças de transmissão de status traduzem-se melhor pelos distintos níveis de crescimento econômico e pelos diferentes setores.

Finalmente, vale ressaltar que a principal contribuição do presente trabalho foi analisar o processo da transmissão intergeracional do status socioeconômico no Brasil, podendo controlar o crescimento econômico quando da entrada dos indivíduos no mercado de trabalho. Isso representa uma abordagem ainda não aplicada à análise de dados brasileiros e, o que é mais importante, permitiu concluir que análises anteriores (BILLS; HALLER, 1984; HALLER, SARAIVA, 1991; NEVES et al., 2007) podem ter se equivocado ao concluírem que o desenvolvimento econômico não apresenta qualquer efeito de redução da transmissão intergeracional do status socioeconômico no Brasil. Vale ressaltar que essa análise só pode ser feita de forma adequada porque foram estimados modelos hierárquicos que representam, para os estudos de transmis- 
são intergeracional de status, a possibilidade de um melhor controle de fatores estruturais, da mesma forma que os modelos logísticos

\section{Referências}

BILLS, D.; HALLER, A. Socioeconomic development and social stratification: reassessing the Brazilian case. Journal of Developing Areas, p. 59-69, 1984.

BLAU, P.; DUNCAN, O. Measuring the status of occupations. In: GRUSKY, D. (Org.). Social stratification: class, race and gender in sociological perspective. Bolder: Westview Press, 1994, p. 204-208.

The American occupational structure. New York: John Wiley \& Sons, 1967.

BOUDON, R. A desigualdade das oportunidades: a mobilidade social nas sociedades industriais. Brasília: Ed. UnB, 1981.

BOURDIEU, P. Economia das trocas simbólicas. 5a ed. São Paulo: Ed. Perspectiva, 2001.

Os três estados do capital cultural. In: NOGUEIRA, M. A. Escritos de educação. Petrópolis, RJ: Vozes, 1998, p. 71-79.

BRESSER-PEREIRA, L. C. Crescimento e desenvolvimento econômico. Disponível em: <http://www.bresserpereira.org.br/ Papers/2007/07.22.CrescimentoDesenvolvimento.Junho19.2008.pdf>. Acesso em: 17 jan. 2011.

BRYK, A. S.; RAUDENBUSH, S. W. Hierarchical linear models: applications and data analysis methods. Newbury Park, London e New Deli: Sage Publications, 1992.

COLLINS, R. Functional conflict theories of education stratification. In: KARABEL, J.; HALSEY, A. Power and ideology in education. Nova York: Oxford University Press, 1977.

The credential society: an historical sociology of education and stratification. New York: Academic Press, 1979.

DAVIS, K.; MOORE, W. E. Alguns princípios condicionais têm possibilitado às análises de mobilidade social a incorporação de dimensões individuais.

de estratificação. In: VELHO, G. (Org.). Estrutura de classes e estratificação social. 4a ed. Rio de Janeiro: Zahar, 1973, p.115-132.

DUNCAN, O. D. Methodological issues in analysis of social mobility. In: SMELSER, N. J.; LIPSET, S. M. Social structure and mobility in economic. Chicago: Aldine, 1966, p. 51-97.

FEATHERMAN, D. L.; HAUSER, R. M. Prestige or socioeconomic scales in the study of occupational achievement? In: GRUSKY, D. (Org.). Social stratification: class, race and gender in sociological perspective. Bolder: Westview Press, 1994, p. 271-272.

FERNANDES, D. C. Estratificação educacional, origem socioeconômica e raça no Brasil: as barreiras da cor. In: PRÊMIO IPEA 40 ANOS - IPEA-CAIXA 2004. Monografias premiadas. Ipea, 2005.

GRUSKY, D. Industrialization and the status attainment process: the thesis of industrialism reconsidered. American Sociological Review, v. 48, p. 494-506, Aug. 1983.

GUEST, A. M. Frontier and urban-industrial explanations of US occupational mobility in late 1800s. Social Science Research, n. 34, p.140-164, 2005.

GUJARATI, D. N. Econometria básica. $3^{\underline{a}}$ ed. São Paulo: Makron Books, 2000.

HALLER, A.; SARAIVA, H. Ascription and status transmition in Brazil. In: SCOVILLE, $J$. Status influences in the third world labor market: caste, gender and custom. New York: Walter de Gruyter, 1991, p. 65-93.

HELAL, D. A dinâmica da estratificação social no setor público brasileiro: meritocracia ou reprodução social? Tese (Doutorado), Universidade Federal de Minas Gerais, Belo Horizonte, 2008. Disponível em: <http://www.bibliotecadigital.ufmg.br/ 
dspace/bitstream/1843/VCSA-7FZV43/1/ tese_vers_o_final.pdf $>$. Acesso em: 17 nov. 2010 .

LINTON, R. O homem: uma introdução à antropologia. 3a ed. São Paulo: Martins Fontes, 1959.

LUKE, D. A. Multilevel modeling. Thousand Oaks, London, New Delhi: Sage Publications, 2004 (A Sage university papers series. Quantitative applications in the social sciences, 143).

NEVES, J. A.; FERNANDES, D. C.; HELAL, D. Region, industry, and intergeracional status transmission in urban Brazil. Population Review, v. 46, n. 1, p. 12-28, 2007.

PARSONS, T. Equality and inequality in modern society, or social stratification revisited. Sociological Inquiry, v. 40, issue 2, p. 13-72, April 1970.

\section{O sistema das sociedades}

modernas. São Paulo: Pioneira, 1974.

PASTORE, J. Desigualdade e mobilidade social no Brasil. São Paulo: T.A. Queiroz Editor, 1979.

PASTORE, J.; SILVA, N. V. Mobilidade social no Brasil. São Paulo: Makron Books, 2000.

RIBEIRO, C. A. C. Estrutura de classe e mobilidade social no Brasil. Bauru, São Paulo: Edusc, 2007.

SCALON, M. C. Mobilidade social no Brasil: padrões e tendências. Rio de Janeiro: Revan, 1999.

SOROKIN, P. Social and cultural mobility. In: GRUSKY, D. (Org.). Social stratification: class, race and gender in sociological perspective. $2^{\underline{a}}$ ed. Bolder: Westview Press, 2001, p. 303-308.

TREIMAN, D. J. Occupational prestige in comparative perspective. In: GRUSKY, D. (Org.). Social stratification: class, race and gender in sociological perspective. $2^{a}$ ed. Bolder: Westview Press, 1994, p. 208-211.

Industrialization and social stratification. Sociological Inquiry, v. 40, issue 2, p. 207-234, April 1970.

TURNER, F. J. The frontier in American history. New York: Henry Holt and Company, 1920. Disponível em: <http://xroads.virginia. edu/ hyper/turner/> . Acesso em: 21 out. 2010.

VILELA, E. M. Imigração internacional e estratificação no mercado de trabalho brasileiro. Tese (Doutorado), Universidade Federal de Minas Gerais, Belo Horizonte, 2008. Disponível em: < http://www.bibliotecadigital.ufmg.br/dspace/bitstream/1843/ VCSA-874KJ4/1/tesefinal_elaine.pdf $>$. Acesso em: 17 nov. 2010.

XAVIER, F. P. Transmissão Intergeracional de status, mercados regionais, setores e crescimento econômico no Brasil. 2011. Tese (Doutorado), Universidade Federal de Minas Gerais, Belo Horizonte, 2011. Disponível em: <http://www.bibliotecadigital. ufmg.br/dspace/bitstream/1843/BUOS$8 \mathrm{H} 7 \mathrm{RCF} / 1 /$ tese_flavia_xavier_revisada.pdf $>$. Acesso em: 16 set. 2011.

\section{Resumen}

\section{Estructura social y transmisión intergeneracional de status: un análisis jerárquico}

Una gran parte de los esfuerzos realizados por los estudios empíricos de los sistemas de estratificación ha correspondido a la investigación de los mecanismos de transmisión de status. Las diferencias organizacionales encontradas entre sectores económicos y las diferencias regionales se insertan, precisamente, en la dimensión estructural de los estudios de estratificación. Así pues, el principal objetivo de este artículo es investigar en qué medida el status ocupacional actual de los individuos analizados se vio afectado por su origen social, teniendo en consideración el momento en el que estos individuos se insertaron en el mercado 
de trabajo y el lugar donde se produjo su inserción. Para ello, fueron utilizadas las bases de la Pesquisa Nacional por Amostra de Domicílios (PNAD7) de 1973, 1982, 1988 e 1996 y se estimaron los modelos jerárquicos que representan, para realizar los estudios de transmisión intergeneracional de status, y analizar la posibilidad de un mejor control de factores estructurales. Se observó que la segmentación del mercado de trabajo, en diferentes sectores económicos, y el nivel de crecimiento económico en Brasil, en diferentes cohortes de entrada de los individuos en el mercado de trabajo, son mecanismos que funcionan como mediadores de la transmisión de status, mientras que en lo que se refiere a la segmentación regional no se presentaron efectos significativos.

Palabras-clave: Transmisión de status. Regiones de Brasil. Sectores económicos. Crecimiento económico. Cohortes de entrada en el mercado de trabajo. Modelos jerárquicos lineales.

\begin{abstract}
Social structure and intergenerational transmission of status: a hierarchical analysis

Empirical studies of stratification systems have concentrated in investigating the mechanisms of transmission of status. Organizational differences found between economic sectors and regional differences fall precisely in the structural dimension of stratification studies. Thus, the main objective of this paper is to investigate to what extent the current occupational status of individuals was affected by their social origin, considering the time when and where these individuals entered the labor market. We used the databases from the National Household Sample Survey (PNAD in Portuguese) of 1973, 1982, 1988 and 1996, and estimated the Hierarchical Models that represent, for studies of intergenerational transmission of status, the possibility of better control of structural factors. We observed that the segmentation of the labor market in different economic sectors and the level of economic growth in Brazil in different cohorts of individuals entering the labor market are mechanisms that function as mediators for the transmission status while regional segmentation had no significant effect.
\end{abstract}

Keywords: Transmission of status. Regions in Brazil. Economic sectors. Economic growth. Cohorts entering the labor market. Hierarchical Linear Models.

Recebido para publicação em 11/11/2011

Aceito para publicação em 12/07/2012

\footnotetext{
7 Investigación Nacional por Muestra de Domicilios
} 
